\title{
Mixing of blackbodies: entropy production and dissipation of sound waves in the early Universe
}

\author{
R. Khatri ${ }^{1}$, R. A. Sunyaev ${ }^{1,2,3}$, and J. Chluba ${ }^{4}$ \\ 1 Max Planck Institut für Astrophysik, Karl-Schwarzschild-Str. 1, 85741 Garching, Germany \\ e-mail: khatri@mpa-garching.mpg.de \\ 2 Space Research Institute, Russian Academy of Sciences, Profsoyuznaya 84/32, 117997 Moscow, Russia \\ 3 Institute for Advanced Study, Einstein Drive, Princeton, New Jersey 08540, USA \\ ${ }^{4}$ Canadian Institute for Theoretical Astrophysics, 60 St George Street, Toronto, ON M5S 3H8, Canada
}

Received 13 May 2012 / Accepted 8 June 2012

\section{ABSTRACT}

\begin{abstract}
Mixing of blackbodies with different temperatures creates a spectral distortion which, at lowest order, is a $y$-type distortion, indistinguishable from the thermal $y$-type distortion produced by the scattering of cosmic microwave background (CMB) photons by hot electrons residing in clusters of galaxies. This process occurs in the radiation-pressure dominated early Universe, when the primordial perturbations excite standing sound waves on entering the sound horizon. Photons from different phases of the sound waves, having different temperatures, diffuse through the electron-baryon plasma and mix together. This diffusion, with the length defined by Thomson scattering, dissipates sound waves and creates spectral distortions in the CMB. Of the total dissipated energy, $2 / 3$ raises the average temperature of the blackbody part of spectrum, while $1 / 3$ creates a distortion of $y$-type. It is well known that at redshifts $10^{5} \lessgtr z \lessgtr 2 \times 10^{6}$, comptonization rapidly transforms $y$-distortions into a Bose-Einstein spectrum. The chemical potential of the Bose-Einstein spectrum is again $1 / 3$ the value we would get if all the dissipated energy was injected into a blackbody spectrum but no extra photons were added. We study the mixing of blackbody spectra, emphasizing the thermodynamic point of view, and identifying spectral distortions with entropy creation. This allows us to obtain the main results connected with the dissipation of sound waves in the early Universe in a very simple way. We also show that mixing of blackbodies in general, and dissipation of sound waves in particular, leads to creation of entropy.
\end{abstract}

Key words. cosmic background radiation - cosmology: theory - early Universe

\section{Introduction}

The cosmic microwave background $(\mathrm{CMB})$ has a spectrum which is close to a blackbody with very high precision. COBE/FIRAS (Fixsen et al. 1996) constrained possible departures from a perfect blackbody to be $\lesssim 10^{-5}$ for $y$-type distortions, and the chemical potential was limited to $|\mu| \lesssim 9 \times 10^{-5}$. This observation immediately has an important consequence: there was a time in the history of the Universe when matter and radiation were in complete thermodynamic equilibrium with each other and any energy release at $z \lesssim 2 \times 10^{6}$ was small.

In addition to being an almost perfect blackbody, the CMB is also isotropic, with anisotropies of less than $10^{-3}$. The dominant component of the anisotropy is the dipole caused by our peculiar motion. The anisotropies other than the dipole are at the level of $10^{-5}$ and originate from the primordial fluctuations imprinted in the initial conditions of the present expanding Universe. The radiation field seen by any observer (or electrons in the hot gas in a cluster of galaxies/early Universe) will thus consist of blackbodies having temperature that differs as a function of observation direction. Scattering by electrons, averaging of the smaller scales within the beam of the telescope or explicit averaging by a cosmologist, will thus inevitably mix these blackbodies together. Mixing of blackbodies was first studied in detail by (Zeldovich et al. 1972) who showed that it creates a $y$-type distortion, indistinguishable from the $y$-type distortion created by interaction of the blackbody photons with hotter electrons (Zeldovich \& Sunyaev 1969). Chluba \& Sunyaev (2004) proved that at lowest order, this result is valid for arbitrary temperature distributions and not just the Gaussian ones. Comptonization of this initial $y$-type distortion converts it into a Bose-Einstein spectrum or a $\mu$-type distortion (Zeldovich \& Sunyaev 1969; Chluba et al. 2012 b) for $y \gtrsim 1$, where the Compton $y$-parameter is defined as the following integral over time $t: y=\int n_{\mathrm{e}} \sigma_{\mathrm{T}} c \frac{k_{\mathrm{B}} T_{\mathrm{e}}}{m_{\mathrm{e}} c^{2}} \mathrm{~d} t, n_{\mathrm{e}}$ is the electron number density, $\sigma_{\mathrm{T}}$ is the Thomson cross section, $c$ is the speed of light, $k_{\mathrm{B}}$ is the Boltzmann's constant, $T_{\mathrm{e}}$ is the electron temperature, and $m_{\mathrm{e}}$ is the mass of electron (Kompaneets 1956; Zeldovich \& Sunyaev 1969). The purpose of this paper is to study the mixing of blackbodies from a statistical physics point of view. Amazingly, using basic thermodynamic relations, we arrive at the main results connected with the dissipation of sound waves in the early Universe due to shear viscosity and thermal conduction in a very simple manner. Some of these aspects are also discussed in Chluba et al. (2012b).

\section{Entropy of a Bose gas}

The entropy density of a general distribution of a Bose gas (photons), which may or may not be in equilibrium, is given by (Landau \& Lifshitz 1980)

$$
\begin{aligned}
S & =\int \mathrm{d} v \frac{8 \pi v^{2}}{c^{3}}[(1+n) \ln (1+n)-n \ln (n)] \\
& =8 \pi\left(\frac{k_{\mathrm{B}} T}{h c}\right)^{3} \int x^{2}[(1+n) \ln (1+n)-n \ln (n)] \mathrm{d} x
\end{aligned}
$$


where $\frac{g d^{3} p}{h^{3}}=\frac{8 \pi v^{2} \mathrm{~d} v}{c^{3}}$ is the density of available states in the frequency interval $\mathrm{d} v$, and for photons the degeneracy $g=2, h$ is Planck's constant, $n=\frac{c^{3} E_{v}}{8 \pi h v^{3}}$ is the photon occupation number and $E_{v}$ is the energy density of photons per unit frequency. We have changed variables to dimensionless frequency $x=$ $h v /\left(k_{\mathrm{B}} T\right)$ defined with respect to a reference temperature $T$ in the second line. For a blackbody spectrum at temperature $T$ with $n=n_{\mathrm{Pl}}(x) \equiv 1 /\left(\mathrm{e}^{x}-1\right)$, we obtain $S_{\mathrm{pl}}=\frac{32 \pi^{5} k_{\mathrm{B}}^{3} T^{3}}{45 c^{3} h^{3}} \equiv \frac{4}{3} a_{\mathrm{R}} T^{3} / k_{\mathrm{B}}$, where the last line defines the radiation constant $a_{\mathrm{R}}$.

Now let us add a small spectral distortion $\delta n$ to the Planck spectrum so that the total occupation number is $n=n_{\mathrm{Pl}}(1+$ $\left.\delta n / n_{\mathrm{Pl}}\right)$, where $\delta n / n_{\mathrm{Pl}} \ll 1$. Expanding the expression for entropy up to first order in $\delta n / n_{\mathrm{Pl}}$ and ignoring the higher order terms, we get $S=S_{\mathrm{pl}}+\delta S$, where $\delta S$ is the entropy density added/subtracted due to the spectral distortion and is given by,

$$
\begin{aligned}
\delta S & \approx 8 \pi\left(\frac{k_{\mathrm{B}} T}{h c}\right)^{3} \int x^{2}\left[\ln \left(1+n_{\mathrm{Pl}}\right)-\ln \left(n_{\mathrm{Pl}}\right)\right] \delta n \mathrm{~d} x \\
& =8 \pi\left(\frac{k_{\mathrm{B}} T}{h c}\right)^{3} \int x^{3} \delta n \mathrm{~d} x \equiv \frac{\delta Q}{k_{\mathrm{B}} T},
\end{aligned}
$$

where $\delta Q$ is the energy density in the distortion. Thus for small distortions, the classic thermodynamical formula for the change in the equilibrium entropy due to addition/subtraction of energy remains valid even in non-equilibrium. We note in particular that, at first order in distortions, the entropy does not depend on the shape of the distortion but only on the total energy density in the distortion. This means that the process of comptonization, which converts an initial $y$-type distortion to a $\mu$-type distortion, in the absence of any additional heating/cooling, does not change the entropy of the photon gas at first order. The factor of $k_{\mathrm{B}}$ in Eq. (2) just converts temperature from Kelvin to energy units to make entropy dimensionless. As an example, we can calculate the entropy produced during cosmological recombination, when $\sim 5$ energetic recombination photons are produced per hydrogen atom (Chluba \& Sunyaev 2006). Adding up the energy in the recombination spectrum (Rubiño-Martín et al. 2008), we get $\delta S_{\text {recombination }} \sim \delta Q_{\text {recombination }} /\left(k_{\mathrm{B}} T_{\mathrm{CMB}}\right) \sim 9 \times 10^{-6} / \mathrm{cm}^{3}$ today, compared with entropy density of $1478 / \mathrm{cm}^{3}$ in the blackbody part of CMB with temperature $T_{\mathrm{CMB}}=2.725 \mathrm{~K}$.

In the rest of the paper we will measure frequency and temperature in energy units and set $c=h=k_{\mathrm{B}}=1$ unless explicitly specified in definitions of other constants.

\section{Mixing of blackbodies}

Since the blackbody radiation is an equilibrium distribution for photons it is described by a single parameter, the temperature $T$. In addition, if we specify a second thermodynamic quantity such as volume $V$, we can calculate any other thermodynamic quantity such as entropy or internal energy. The spectrum is described by the well known Planck function, and the energy density per unit frequency and total energy density integrated over frequency can be written as,

$$
\begin{aligned}
E_{v} & =8 \pi v^{3} \frac{1}{\mathrm{e}^{\frac{v}{T}}-1} \\
E & =a_{\mathrm{R}} T^{4} .
\end{aligned}
$$

The number density of photons is given by $N=b_{\mathrm{R}} T^{3}$ where $b_{\mathrm{R}}=\frac{16 \pi k_{\mathrm{B}}{ }^{3} \zeta(3)}{c^{3} h^{3}}, \zeta$ is the Riemann zeta function with $\zeta(3) \approx$ 1.20206. We can also calculate the entropy density and it is given by $S=4 E /(3 T)=(4 / 3) a_{\mathrm{R}} T^{3}$. For the CMB with $T_{\mathrm{CMB}}=$ $2.725 \mathrm{~K} \pm 1 \mathrm{mK}$ (Fixsen \& Mather 2002) we have energy density $E_{\mathrm{CMB}}=0.26 \mathrm{eV} / \mathrm{cm}^{3}$, number density $N_{\mathrm{CMB}}=411 / \mathrm{cm}^{3}$ and entropy density $S_{\mathrm{CMB}}=1478 / \mathrm{cm}^{3}$. For simplicity, we will consider mixing of two blackbodies below, but the derivation and the results are trivially generalized to an ensemble of arbitrary number of blackbodies by just replacing the average of quantities over two blackbodies with the appropriate average over the whole ensemble.

\section{1. y-type distortion}

If we mix blackbody spectra with different temperature, the resultant spectrum is not blackbody and at lowest order the distortion is given by a $y$-type spectrum (Zeldovich et al. 1972) ${ }^{1}$. This can be seen immediately by Taylor expanding the photon intensity or equivalently the occupation number $n$ of a blackbody at a temperature $T+\Delta T$ about the average temperature $T$ and take the average (ensemble or spatial) keeping terms up to second order in $\Delta T / T$,

$$
\begin{aligned}
& \left\langle n_{\mathrm{Pl}}(T+\Delta T)\right\rangle \equiv\left\langle\frac{1}{\mathrm{e}^{\frac{v}{(T+\Delta T)}}-1}\right\rangle \\
\approx & \left\langle n_{\mathrm{Pl}}(T)+\ln \left[1+\frac{\Delta T}{T}\right] \frac{\partial n_{\mathrm{Pl}}}{\partial \ln [T]}+\frac{1}{2}\left(\ln \left[1+\frac{\Delta T}{T}\right]\right)^{2} \frac{\partial^{2} n_{\mathrm{Pl}}}{\partial(\ln [T])^{2}}\right\rangle \\
= & n_{\mathrm{Pl}}(T)+\left(\left\langle\frac{\Delta T}{T}\right\rangle+\left\langle\left(\frac{\Delta T}{T}\right)^{2}\right\rangle\right) T \frac{\partial n_{\mathrm{Pl}}}{\partial T}+\frac{1}{2}\left\langle\left(\frac{\Delta T}{T}\right)^{2}\right\rangle T^{4} \frac{\partial}{\partial T} \frac{1}{T^{2}} \frac{\partial n_{\mathrm{Pl}}}{\partial T} \\
= & n_{\mathrm{Pl}}\left[T\left(1+\left\langle\left(\frac{\Delta T}{T}\right)^{2}\right\rangle\right)\right]+\frac{1}{2} Y(x)\left\langle\left(\frac{\Delta T}{T}\right)^{2}\right\rangle,
\end{aligned}
$$

where we have used $\left\langle\frac{\Delta T}{T}\right\rangle \equiv 0$. The first term in the last line is simply a blackbody with temperature $T_{\text {new }}=T\left[1+(\Delta T / T)^{2}\right]>$ $T$, and

$Y(x)=\frac{x \mathrm{e}^{x}}{\left(\mathrm{e}^{x}-1\right)^{2}}\left(x \frac{\mathrm{e}^{x}+1}{\mathrm{e}^{x}-1}-4\right)$

is the $y$-type spectrum with $x=v / T$ (Zeldovich \& Sunyaev 1969) with the magnitude of distortion given by $y_{Y}=\frac{1}{2}\left\langle\left(\frac{\Delta T}{T}\right)^{2}\right\rangle$. The average spectrum with $y$-type distortion for average of two blackbodies with temperature $T \pm \Delta T$ is shown in Fig. 1 . Figure 2 shows the same spectra but in terms of the effective temperature defined by $n(v)=1 /\left(\mathrm{e}^{v / T_{\text {eff }}(v)}-1\right)$, which for small distortions can be written, at linear order, as $T_{\text {eff }}(x)=$ $T+T\left[\left(1-\mathrm{e}^{-x}\right) / x\right] \delta n / n$. We note that in the Rayleigh-Jeans limit, intensity is proportional to temperature, and thus the average of blackbody spectra just gives the spectrum at average temperature $T$, and $T_{\text {eff }} \rightarrow T$. An important property of $y$-type distortion is that it represents pure redistribution of photons in the spectrum due to addition of energy but conserves photon number. Thus $\int x^{2} Y(x) \mathrm{d} x=0$ and the above mentioned decomposition of the spectrum into a blackbody part and $y$-distortion part is unique and independent of gauge/reference frame, at order $\delta n / n$, in the following sense: the constraint $\int x^{2} \delta n(x) \mathrm{d} x=0$ on the spectral distortion $\delta n$ fixes the reference temperature of the blackbody part of the spectrum used to define the variable $x$ and $\delta n$ as a function of the variable $x$ is gauge independent ${ }^{2}$.

1 We discuss different processes which can lead to the mixing of blackbodies in the following sections.

${ }^{2}$ In fact $\delta n(x)$ is also invariant to change in the reference temperature happening in the mixing of blackbodies, $\delta n(x)=\delta n\left(x_{\text {new }}\right)+O\left([\delta n / n]^{2}\right)$, where $x_{\text {new }}=v / T_{\text {new }}$. 
R. Khatri et al.: Mixing of blackbodies

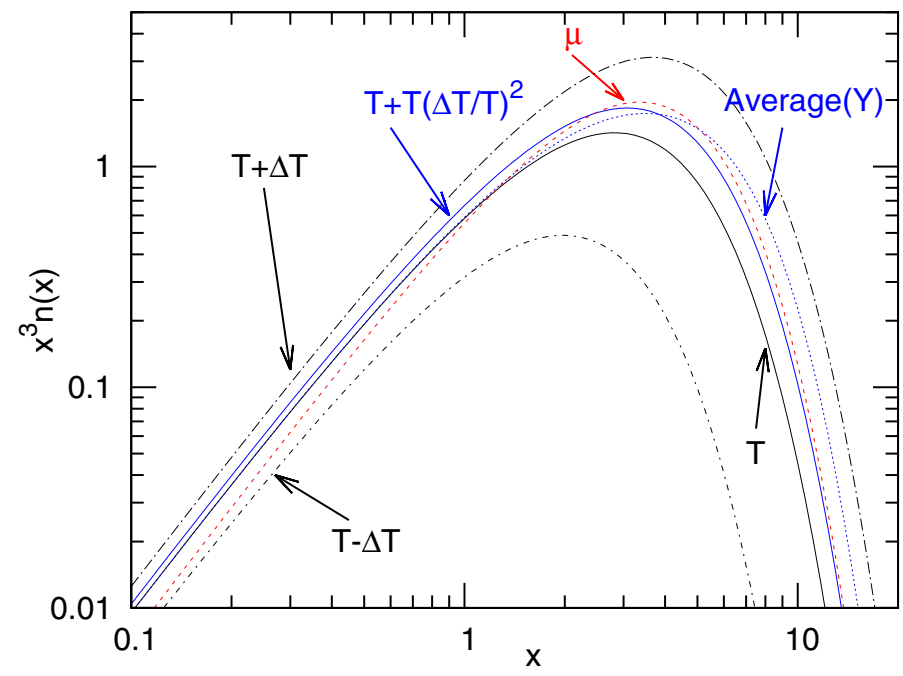

Fig. 1. Average of blackbodies of temperature $T+\Delta T$ and $T-\Delta T$ creates a new spectrum marked "Average $(Y)$ " which is different from blackbody spectrum marked with temperature $T$. The average spectrum is just the usual $y$-type distortion with respect to a new blackbody at temperature $T\left[1+(\Delta T / T)^{2}\right]$, with the two crossing at $x=3.83$. At redshifts $z \gtrsim 10^{5}$ the average spectrum will comptonize to Bose-Einstein spectrum marked $\mu$ above. All three spectra, $T\left[1+(\Delta T / T)^{2}\right]$, Average $(Y)$, and $\mu$, have the same number density of photons. Average/y-type spectrum and Bose-Einstein/ $\mu$-type spectrum also have the same energy density which is greater than the energy density in the blackbody spectrum with temperature $T\left[1+(\Delta T / T)^{2}\right]$ by $1 / 3$ of the initial energy density excess over that of blackbody with temperature $T$. We have used linear order formulae to calculate the $y$ and $\mu$ distortions in the figure but used a large value of $\Delta T / T$ to make differences between different curves visible.

Without loss of generality, let us consider the superposition of two blackbody spectra with temperatures $T_{1}=T+\Delta T$ and $T_{2}=T-\Delta T$, with average temperature $T$. The average initial energy density, number density and entropy density of the two blackbodies is

$$
\begin{aligned}
& E_{\text {initial }}=\frac{1}{2} a_{\mathrm{R}}\left(T_{1}^{4}+T_{2}^{4}\right) \approx a_{\mathrm{R}} T^{4}\left[1+6\left(\frac{\Delta T}{T}\right)^{2}\right]>a_{\mathrm{R}} T^{4} \\
& N_{\text {initial }}=\frac{1}{2} b_{\mathrm{R}}\left(T_{1}^{3}+T_{2}^{3}\right) \approx b_{\mathrm{R}} T^{3}\left[1+3\left(\frac{\Delta T}{T}\right)^{2}\right]>b_{\mathrm{R}} T^{3} \\
& S_{\text {initial }}=\frac{1}{2} \frac{4 a_{\mathrm{R}}}{3}\left(T_{1}^{3}+T_{2}^{3}\right) \approx \frac{4 a_{\mathrm{R}}}{3} T^{3}\left[1+3\left(\frac{\Delta T}{T}\right)^{2}\right]>\frac{4 a_{\mathrm{R}}}{3} T^{3} .
\end{aligned}
$$

We can calculate the final temperature of a blackbody having the same number density of photons as the initial average $N_{\text {initial }}$

$T_{\text {final }}=\left(\frac{N_{\text {initial }}}{b_{\mathrm{R}}}\right)^{1 / 3} \approx T\left[1+\left(\frac{\Delta T}{T}\right)^{2}\right]$

This is exactly the temperature, $T_{\text {new }}$, of the blackbody we got by averaging the intensity in Eq. (4). Thus all the initial photons go into creating a blackbody with a higher temperature. The entropy density of this new blackbody is also identical to the initial average entropy density $S_{\text {initial }}$ because number density and entropy density have the same $T^{3}$ temperature dependence. The energy density of the new blackbody is however given by,

$E_{\text {final }}=a_{\mathrm{R}} T_{\text {final }}^{4} \approx a_{\mathrm{R}} T^{4}\left[1+4\left(\frac{\Delta T}{T}\right)^{2}\right]<E_{\text {initial }}$.

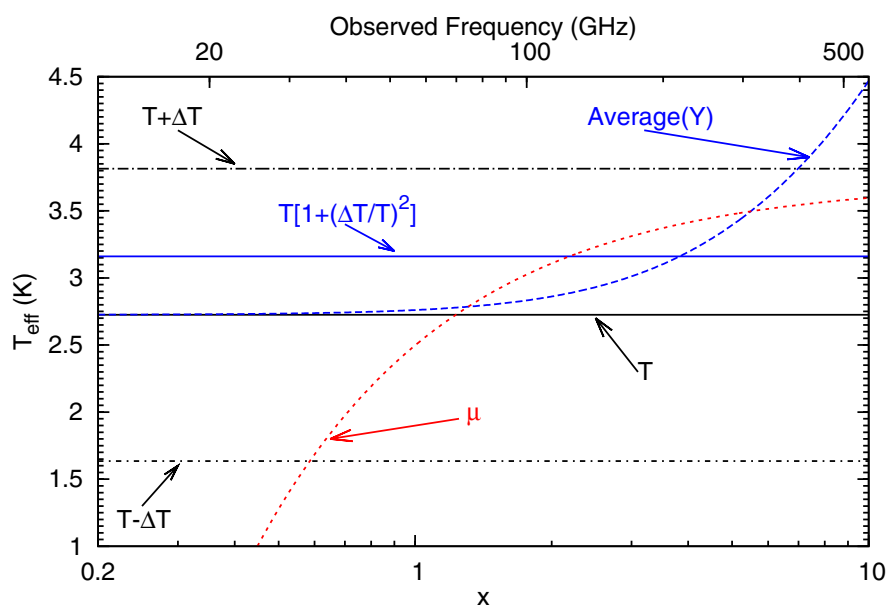

Fig. 2. Same as Fig. 1 but effective temperature $T_{\text {eff }}(v)$ defined by $n(v)=1 /\left(\mathrm{e}^{v / T_{\text {eff }}(v)}-1\right)$ is plotted. We have used linear order formulae to calculate the $y$ and $\mu$ distortions in the figure but used a large value of $\Delta T / T=0.4$ to make differences between different curves visible. Average temperature $T=2.725 \mathrm{~K}$. For Bose-Einstein spectrum we thus have, at linear order, $T_{\mathrm{eff}}(x)=T_{\mathrm{BE}}-T \mu / x$. For a general distortion $\delta n / n$ (including $y$-type ) we have $T_{\text {eff }}(x)=T+T\left[\left(1-\mathrm{e}^{-x}\right) / x\right] \delta n / n$.

In fact we find

$E_{\text {final }}-a_{\mathrm{R}} T^{4}=2 / 3\left(E_{\text {initial }}-a_{\mathrm{R}} T^{4}\right)$.

This result can also be obtained directly by multiplying Eq. (4) by $v^{3}$ and integrating over frequency. Equation (4) also shows that the rest of the initial energy, equal to

$1 / 3\left(E_{\text {initial }}-a_{\mathrm{R}} T^{4}\right)=2\left(\frac{\Delta T}{T}\right)^{2} a_{\mathrm{R}} T^{4} \propto \frac{1}{2}\left(\frac{\Delta T}{T}\right)^{2} \int \mathrm{d} x x^{3} Y(x)$,

goes to the $y$-distortion with the magnitude of the distortion given by $y_{Y}=\frac{1}{2}\left(\frac{\Delta T}{T}\right)^{2}$. These results were recently obtained in Chluba et al. (2012b) using the Boltzmann equation. It is well known (Zeldovich \& Sunyaev 1969) that $y$-distortion of magnitude $y_{Y}$ decreases the brightness temperature of radiation in the Rayleigh-Jeans part of the spectrum by an amount $-2 y_{Y}$ which is equal to $\left(\frac{\Delta T}{T}\right)^{2}$ for the mixing of blackbodies considered here. But we have also increased the magnitude of the brightness temperature by same amount in the blackbody part of the spectrum, resulting in the brightness temperature in the Rayleigh-Jeans part for the total spectrum which is equal to the average temperature $T$ of the initial blackbodies, as shown in Figs. 1 and 2. We can calculate the additional entropy in the final spectrum resulting from the mixing of blackbodies using Eq. (2),

$\Delta S=\frac{\Delta E}{T} \approx 2 a_{\mathrm{R}} T^{3}\left(\frac{\Delta T}{T}\right)^{2}$,

where $\Delta E=1 / 3\left(E_{\text {initial }}-a_{\mathrm{R}} T^{4}\right)$ is the energy in spectral distortion, i.e. deviation from the blackbody spectrum.

To summarize, mixing/averaging of blackbodies leads to a new spectrum which has a blackbody part at a temperature which is higher than the average temperature of initial blackbodies by $\Delta T_{\text {new }}=T(\Delta T / T)^{2}$. This new blackbody has the same entropy as initial average entropy and the same number of photons but less energy. The remaining energy density appears as a $y$-type distortion, which is just a redistribution of the photons of the 
new blackbody spectrum. The $y$-distortion part can be identified with the increase in the entropy of the system (Eq. (11)), which is expected as there is an increase in disorder of the system.

\section{2. $\mu$-type distortion}

We saw in the previous section that it is impossible to create a blackbody spectrum by mixing/averaging blackbodies of different temperatures. The reason is that there is too much energy density for the given number density of photons. However we can still create a Bose-Einstein spectrum with a non zero chemical potential $\mu$, which is the spectrum we would get if the photons could again reach equilibrium while conserving energy and number. It is straightforward to calculate the temperature and chemical potential of the Bose-Einstein spectrum, $n_{\mathrm{BE}}=1 /\left(\mathrm{e}^{v / T_{\mathrm{BE}}+\mu}-1\right)$, by equating the initial average photon number $N_{\text {initial }}$ and energy density $E_{\text {initial }}$ to the photon number $N_{\mathrm{BE}}$ and energy density $E_{\mathrm{BE}}$ in a Bose-Einstein spectrum, as is done in case of heating of CMB without adding additional photons (Illarionov \& Sunyaev 1975). In the limit of small chemical potential, $\mu \ll 1$, with $T_{\mathrm{BE}}=T_{\text {new }}\left(1+t_{\mathrm{BE}}\right) \approx$ $T\left(1+t_{\mathrm{BE}}+[\Delta T / T]^{2}\right), t_{\mathrm{BE}} \ll 1$ we have (Illarionov \& Sunyaev $1975)^{3}$

$$
\begin{aligned}
E_{\mathrm{BE}} & \approx a_{\mathrm{R}} T_{\mathrm{BE}}^{4}(1-1.1106 \mu) \\
& \approx a_{\mathrm{R}} T^{4}\left(1+4 t_{\mathrm{BE}}+4\left(\frac{\Delta T}{T}\right)^{2}-1.1106 \mu\right) \\
& =E_{\text {initial }} \approx a_{\mathrm{R}} T^{4}\left[1+6\left(\frac{\Delta T}{T}\right)^{2}\right],
\end{aligned}
$$

$N_{\mathrm{BE}} \approx b_{\mathrm{R}} T_{\mathrm{BE}}^{3}(1-1.3684 \mu)$

$$
\begin{aligned}
& \approx b_{\mathrm{R}} T^{3}\left(1+3 t_{\mathrm{BE}}+3\left(\frac{\Delta T}{T}\right)^{2}-1.3684 \mu\right) \\
& =N_{\text {initial }} \approx b_{\mathrm{R}} T^{3}\left[1+3\left(\frac{\Delta T}{T}\right)^{2}\right] .
\end{aligned}
$$

The second equation, $N_{\mathrm{BE}}=N_{\text {initial }}$, directly gives us the relation $t_{\mathrm{BE}}=1.3684 \mu / 3=\mu / 2.19$. Solving the system of equations for $t_{\mathrm{BE}}$ and $\mu$ in terms of $\Delta T / T$, we get

$$
\begin{aligned}
\mu & =1.4\left[\frac{1}{3}\left(E_{\text {initial }}-a_{\mathrm{R}} T^{4}\right)\right]=2.8\left(\frac{\Delta T}{T}\right)^{2} \\
t_{\mathrm{BE}} & =\frac{\mu}{2.19}=1.278\left(\frac{\Delta T}{T}\right)^{2}
\end{aligned}
$$

this is exactly the result we will get if we add energy $\Delta E=$ $\frac{1}{3}\left(E_{\text {initial }}-a_{\mathrm{R}} T^{4}\right)$ to a blackbody with temperature $T_{\text {new }}$ while conserving the photon number. We can thus write the deviation of the $\mu$-type spectrum from the blackbody with temperature $T_{\text {new }}$ :

$$
\frac{n_{\mathrm{BE}}-n_{\mathrm{Pl}}\left(T_{\text {new }}\right)}{n_{\mathrm{Pl}}\left(T_{\text {new }}\right)}=\left(t_{\mathrm{BE}} x-\mu\right) \frac{\mathrm{e}^{x}}{\mathrm{e}^{x}-1} .
$$

The $\mu$-type spectrum resulting from the average of two blackbodies is also shown in Figs. 1 and 2. An important point to note here is that the Bose-Einstein spectrum is uniquely fixed

\footnotetext{
${ }^{3}$ Using $6 \zeta(3) / I_{3} \approx 1.1106$ and $\pi^{2} /\left(3 I_{2}\right) \approx 1.3684$, where $\zeta$ is the Riemann zeta function with $\zeta(3) \approx 1.20206$, and $I_{3}=\int x^{3} n_{\mathrm{Pl}}(x) \mathrm{d} x=$ $\pi^{4} / 15, I_{2}=\int x^{3} n_{\mathrm{Pl}}(x) \mathrm{d} x=2 \zeta(3)$.
}

by energy density and number density constraints. In particular, the value of the chemical potential $\mu$ is independent of any reference temperature we may choose to define the dimensionless variable $x$. This is, of course, the same value of $\mu$ we would get if we just comptonize the $y$-type distortion of the previous section. The Bose-Einstein spectrum and the chemical potential has however more fundamental origins in statistical physics, compared to the $y$-type distortion, the shape of which originates in the Compton scattering process or sum of Planck spectra. The $\mu$-type results of this section can thus be considered as the basis for our definitions of the spectral distortion as pure redistribution of photons, and in particular of the 2:1 division of initial energy in temperature perturbations into a blackbody part and a spectral distortion part. Further justification is provided by the fact that with this definition, the spectral distortion can also be identified with the entropy production.

One important difference from the heating of CMB usually considered, for example, in clusters of galaxies or due to decay of particles in the early Universe, is that in mixing of blackbodies, we are also adding photons (compared to a blackbody at initial average temperature). The additional photons are able to create a new blackbody at a higher temperature, which is impossible if the photon number density is kept constant.

Although we have derived the above formulae by considering only two blackbodies, they are applicable to an ensemble with arbitrary number of blackbodies by just replacing the average over two blackbodies $(\Delta T / T)^{2}$ with the average over the ensemble, $\left\langle(\Delta T / T)^{2}\right\rangle$.

\section{Example: Mixing of the blackbodies in the observed CMB sky}

One obvious and cleanest source of blackbodies of different temperatures is the CMB sky. The temperature in different directions in the sky differs by $\sim 10 \mu \mathrm{K}$ (Bennett et al. 1996; Komatsu et al. 2011) corresponding to the $10^{-5}$ spatial fluctuations in the energy density of radiation/matter in the early Universe before recombination ${ }^{4}$. Thus any telescope, due to finite width of its beam, looking at the microwave sky will inevitably mix the spectra of blackbodies of different temperature within the beam (Chluba \& Sunyaev 2004). In addition we may explicitly average the intensity over the whole sky to achieve higher sensitivity and precision in the measurement of CMB spectrum as is done, for example, by COBE (Fixsen et al. 1996) and in the proposed experiment PIXIE (Kogut et al. 2011). The (angular) average amount of energy in CMB anisotropies is given by (Chluba \& Sunyaev 2004),

$\frac{\Delta E}{E_{\gamma}}=6\left\langle\left(\frac{\Delta T}{T}\right)^{2}\right\rangle=\frac{6}{4 \pi} \sum_{\ell=2}^{\infty}(2 \ell+1) C_{\ell} \approx 9.6 \times 10^{-9}$,

where $E_{\gamma}$ is the energy density of CMB photons. One third of this energy creates a $y$-distortion of magnitude $y_{Y}=\frac{1}{2}\left\langle\left(\frac{\Delta T}{T}\right)^{2}\right\rangle=$ $\frac{1}{12} \frac{\Delta E}{E_{\gamma}}=8 \times 10^{-10}$. The measured temperature from averaged intensity is also higher than the averaged temperature $T_{\mathrm{CMB}}$ by $T_{\mathrm{CMB}}\left\langle\left(\frac{\Delta T}{T}\right)^{2}\right\rangle=4.4 \mathrm{nK}$ accounting for the remaining $2 / 3$ of energy in anisotropies. The increase in entropy in this mixing is $S_{\mathrm{CMB}} \times \frac{3}{2}\left\langle\left(\frac{\Delta T}{T}\right)^{2}\right\rangle=3.5 \times 10^{-6} / \mathrm{cm}^{3}$.

${ }^{4}$ On very small scales the fluctuations are considerably smaller since these fluctuations were erased due to diffusion of photons before and during recombination (Silk damping). 


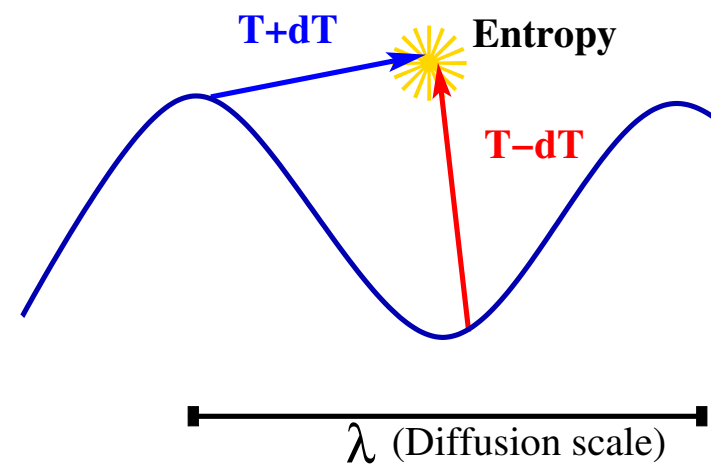

Fig. 3. Cartoon picture of mixing of blackbodies: photons from different phases of sound wave mix and create spectral distortion/entropy.

In the above estimate we ignored the dipole which has been measured by COBE and WMAP (Bennett et al. 1996; Jarosik et al. 2011) to have an amplitude equal to $3.355 \pm 0.008 \mathrm{mK}$ corresponding to our peculiar motion $v=1.23 \times 10^{-3}$. The average power from dipole is then $3 C_{1} /(4 \pi)=v^{2} / 3$. The resulting $y$ distortion is $v^{2} / 6=2.5 \times 10^{-7}$ and increase in monopole temperature is $v^{2} / 3=1.4 \mu \mathrm{K}$. The increase in entropy from the mixing of the CMB dipole on our sky is $\Delta S=1.1 \times 10^{-3} / \mathrm{cm}^{3}$.

\section{Application: dissipation of sound waves in the early Universe}

Before recombination, we have a tightly coupled plasma of radiation-electrons-baryons in the early Universe. At high redshifts, both the energy density and pressure in the plasma are dominated by radiation while at low redshifts, but before recombination, the baryon energy density becomes important, although pressure is still dominated by radiation. Sound speed in this relativistic plasma is therefore $1 / \sqrt{3}$ and Jeans scale or sound horizon is particle horizon $/ \sqrt{3}$. Primordial perturbation on scales smaller than the Jeans scale or sound horizon therefore oscillate setting up standing sound waves (Lifshitz 1946; see also Sunyaev \& Zeldovich 1970b). Although the photon mean free path due to Compton scattering on electrons is very small, they are still able to traverse considerable distance since the big bang, performing a random walk among the electrons. This diffusion and mixing of photons as a result of Thomson scattering erases the sound waves on scales corresponding to the diffusion scale (and smaller). Macroscopically, the dissipation of sound waves can be identified as due to the shear viscosity and thermal conduction in the relativistic fluid composed of baryons, electrons and photons. The damping of sound waves on small scales due to thermal conduction was pointed out by Lifshitz (1946) and first calculated by (Silk 1968) and is known as Silk damping. At high redshifts $(z \gg 693)$ when the energy density of the plasma is also dominated by radiation, shear viscosity is more important than thermal conductivity and was calculated by Peebles \& Yu (1970), later Kaiser (1983) included the effect of polarization (see also Weinberg 1971). The resulting spectral distortions in CMB were considered by Sunyaev \& Zeldovich (1970a); Daly (1991); Hu et al. (1994a). Sunyaev \& Zeldovich (1970a) demonstrated that the upper limit to the $\mu$-type distortions allows us to constrain the amplitude of the primordial fluctuations, which were completely damped in the CMB (on small scales), and are today in the unobservable part of the matter/CMB power spectrum.

Microscopically, diffusion of photons mixes photons from different phases of sound waves which have different temperatures. This is shown schematically in Fig. 3. This implies that locally a $y$-type distortion is created, which quickly comptonizes to a $\mu$-type distortion at $z \gtrsim 10^{5}$, as calculated in the previous sections. The dissipation of sound waves is best understood in Fourier space, denoting Fourier transform of $\frac{\Delta T}{T}(\boldsymbol{x}, \hat{\boldsymbol{n}})$ with $\Theta(\boldsymbol{k}, \hat{\boldsymbol{n}}), \hat{\boldsymbol{n}}$ is the photon direction ${ }^{5}, \boldsymbol{x}$ is the comoving coordinate and $\boldsymbol{k}$ is the comoving wavenumber (see also Chluba et al. 2012b),

$$
\begin{aligned}
\left.\frac{\Delta E}{E_{\gamma}}\right|_{\text {acoustic }} & =6\left\langle\left(\frac{\Delta T}{T}\right)^{2}\right\rangle \\
& =6 \int \frac{\mathrm{d}^{3} k}{(2 \pi)^{3}} \mathrm{e}^{i \boldsymbol{k} \cdot \boldsymbol{x}} \int \frac{\mathrm{d}^{3} k^{\prime}}{(2 \pi)^{3}}\left\langle\Theta\left(\boldsymbol{k}^{\prime}, \hat{\boldsymbol{n}}\right) \Theta\left(\boldsymbol{k}-\boldsymbol{k}^{\prime}, \hat{\boldsymbol{n}}\right)\right\rangle \\
& =6 \int \frac{k^{2} \mathrm{~d} k}{2 \pi^{2}} P_{i}(k)\left[\sum_{\ell=0}^{\infty}(2 \ell+1) \Theta_{\ell}^{2}(k)\right]
\end{aligned}
$$

where we have expanded the temperature perturbation transfer functions in Legendre polynomial basis, $\Theta(\hat{\boldsymbol{n}} \cdot \hat{\boldsymbol{k}}, k)=$ $\sum_{\ell}(-i)^{\ell}(2 \ell+1) \mathcal{P}_{\ell}(\hat{\boldsymbol{n}} \cdot \hat{\boldsymbol{k}}) \Theta_{\ell}(k), \hat{\boldsymbol{n}}$ is the photon direction, $\boldsymbol{k}$ is the unit vector along the Fourier mode which is parallel to the electron peculiar velocity in linear theory. This transformation is possible since in first order perturbation theory the photon transfer functions depend only on $\hat{\boldsymbol{n}} \cdot \hat{\boldsymbol{v}}$ or in Fourier space on $\hat{\boldsymbol{n}} \cdot \hat{\boldsymbol{k}}$ and not on $\hat{\boldsymbol{n}}$ and $\hat{\boldsymbol{k}}$ separately. $P_{i}(k)$ is the power spectrum of initial curvature perturbations $\left(\zeta_{i}\right)$ with respect to which the transfer functions $\Theta_{\ell}$ are calculated. We have also used homogeneity and isotropy of the Universe to carry out one of the integrals and angular part of the second integral. Cosmological perturbations, in particular monopole and dipole depend on the choice of gauge. We will use conformal Newtonian gauge from now on. The spectral distortions defined as pure redistribution of photons, for example the $y$ distortions we are considering, are however gauge independent.

Before cosmological recombination starts with helium recombination at $z \sim 6000$, electrons/baryons and radiation are tightly coupled and $\ell>2$ modes can be neglected. Most of the energy of sound waves is in monopole and dipole and can be calculated using relation between monopole and dipole in the tight coupling regime, $\Theta_{1} \approx \Theta_{0} / \sqrt{3}$ (using sound speed $c_{\mathrm{s}} \approx 1 / \sqrt{3}$ ),

$$
\begin{aligned}
\left.\frac{\Delta E}{E_{\gamma}}\right|_{\text {acoustic }} & =6 \int \frac{k^{2} \mathrm{~d} k}{2 \pi^{2}} P_{i}(k)\left[\Theta_{0}^{2}+3 \Theta_{1}^{2}\right] \\
& \approx \int \frac{k^{2} \mathrm{~d} k}{2 \pi^{2}} P_{i}(k)\left[12 \Theta_{0}^{2}\right]
\end{aligned}
$$

This result is $9 / 4$ times the estimate used in Sunyaev \& Zeldovich (1970a); Hu et al. (1994a); Khatri et al. (2012) where it was also assumed that all of the energy in sound waves gives rise to spectral distortions. As discussed above, $1 / 3$ of this energy, when dissipated due to mixing of blackbodies, sources spectral distortions which are created as $y$-type but rapidly comptonize to $\mu$-type distortions or Bose-Einstein spectrum at high redshifts $z \gtrsim 10^{5}$. The remaining $2 / 3$ of the dissipated energy raises the average temperature of $\mathrm{CMB}$ which is not directly observable. Thus the correct result for distortions differs from earlier estimates only by a factor of $1 / 3 \times 9 / 4=3 / 4$ (Chluba et al. 2012b).

\footnotetext{
5 Bold letters denote vectors, bold letters with hat denote unit vectors and normal letters denote magnitude of the vector.
} 
At redshifts $10^{5} \lesssim z \lesssim 2 \times 10^{6}$, the average $\mu$ distortion therefore increases at a rate,

$$
\begin{aligned}
\frac{\mathrm{d} \mu}{\mathrm{d} t} & =-\left.1.4 \frac{\mathrm{d}}{\mathrm{d} t} \frac{1}{3} \frac{\Delta E}{E_{\gamma}}\right|_{\text {acoustic }} \\
& \approx-\frac{\mathrm{d}}{\mathrm{d} t} \int \frac{k^{2} \mathrm{~d} k}{2 \pi^{2}} P_{i}(k)\left[5.6 \Theta_{0}^{2}\right]
\end{aligned}
$$

and rate of increase of entropy density is given by,

$$
\begin{aligned}
\frac{\mathrm{d} \Delta S / S}{\mathrm{~d} t} & =-\left.\frac{1}{4} \frac{\mathrm{d}}{\mathrm{d} t} \frac{\Delta E}{E_{\gamma}}\right|_{\text {acoustic }} \\
& \approx-\frac{\mathrm{d}}{\mathrm{d} t} \int \frac{k^{2} \mathrm{~d} k}{2 \pi^{2}} P_{i}(k)\left[3 \Theta_{0}^{2}\right]
\end{aligned}
$$

The above rates are easily calculated using analytic tight coupling solutions given by Hu \& Sugiyama (1995) and for power spectrum with constant scalar index it is possible to do the time derivatives and integral analytically. In particular the expressions presented in Khatri et al. (2012) for $\mu$ type distortions remain valid after multiplication by a factor of $3 / 4$. The $y$ - type distortions at $z \lesssim 10^{4}$ require inclusion of additional modifications due to breakdown of tight coupling during recombination, second order Doppler effect, and higher order temperature anisotropies, which were derived in Chluba et al. (2012b) using second order Boltzmann equation.

We can use the first order Boltzmann equation, $\mathrm{d} \Theta / \mathrm{d} t=\Theta_{0}-$ $\frac{1}{2}\left[\Theta_{2}+\Theta_{0}^{\mathrm{P}}+\Theta_{2}^{\mathrm{P}}\right] \mathcal{P}_{2}(\hat{\boldsymbol{n}} \cdot \hat{\boldsymbol{k}})-\Theta(\hat{\boldsymbol{n}} \cdot \hat{\boldsymbol{k}})-\mathrm{i} v \mathcal{P}_{1}(\hat{\boldsymbol{n}} \cdot \hat{\boldsymbol{k}})$, to calculate the time derivative of Eq. (16). Taking into account that only $1 / 3$ of dissipated energy leads to spectral distortions, and requiring that the dipole/velocity term is gauge invariant gives us the full result ${ }^{6}$,

$$
\begin{aligned}
\left.\frac{\mathrm{d}}{\mathrm{d} t} \frac{\Delta E}{E_{\gamma}}\right|_{\text {distortion }} & =-4\left\langle\frac{\Delta T}{T} \frac{\mathrm{d}}{\mathrm{d} t} \frac{\Delta T}{T}\right\rangle \\
\stackrel{\text { ignore metric perturbations }}{\longrightarrow} & 4 n_{\mathrm{e}} \sigma_{\mathrm{T}} \int \frac{k^{2} \mathrm{~d} k}{2 \pi^{2}} P_{i}(k)\left[\Theta_{1}\left(3 \Theta_{1}-v\right)\right. \\
+ & \left.\frac{9}{2} \Theta_{2}^{2}-\frac{1}{2} \Theta_{2}\left(\Theta_{2}^{\mathrm{P}}+\Theta_{0}^{\mathrm{P}}\right)+\sum_{\ell \geq 3}(2 \ell+1) \Theta_{\ell}^{2}\right] \\
\stackrel{\text { impose gauge invariance }}{\longrightarrow} & 4 n_{\mathrm{e}} \sigma_{\mathrm{T}} \int \frac{k^{2} \mathrm{~d} k}{2 \pi^{2}} P_{i}(k)\left[\frac{\left(3 \Theta_{1}-v\right)^{2}}{3}\right. \\
+ & \left.\frac{9}{2} \Theta_{2}^{2}-\frac{1}{2} \Theta_{2}\left(\Theta_{2}^{\mathrm{P}}+\Theta_{0}^{\mathrm{P}}\right)+\sum_{\ell \geq 3}(2 \ell+1) \Theta_{\ell}^{2}\right],
\end{aligned}
$$

where $\boldsymbol{v}_{\mathrm{e}}(k) \equiv-\mathrm{i} \hat{\boldsymbol{k}} v(k)$ is the transfer function of peculiar velocity of baryons/electrons and $\Theta_{\ell}^{\mathrm{P}}$ denote polarization multipole moments. This expression was first derived by Chluba et al. (2012b) using the second order Boltzmann equation, which automatically takes care of the gauge independence and metric perturbations. Using that the spectral distortions (defined as a pure redistribution of photons) are gauge invariant, together with the fact that the only physical mechanism operating here is the mixing of blackbodies, allows us to derive the full result without referring to the second order Boltzmann equation and using only

\footnotetext{
${ }^{6}$ We have ignored the gravitational potential/metric perturbations since they cannot create spectral distortions. They do cancel out explicitly in the second order Boltzmann equation (Chluba et al. 2012b). Gravitational potential/metric perturbations do contribute to the average CMB temperature but this effect is unobservable.
}

the well studied first order Boltzmann equation. The identification of a symmetry in the problem, i.e. gauge invariance, allows us to derive very simply the results of the extensive calculation of Chluba et al. (2012b) corresponding to the average spectral distortions in $\mathrm{CMB}$ created by the dissipation of sound waves. Using the Boltzmann equation, on the other hand, also allows Chluba et al. (2012b) to obtain new results on anisotropies of the spectral distortions, and we refer to that work for a detailed discussion.

We note that with our definitions, all the photon transfer functions and $v$ are real quantities. We have also the introduced multipole moments of degree of polarization, $\Theta^{\mathrm{P}}$, defined in the same way as the temperature multipole moments ${ }^{7}$. The polarization terms are coming directly from the first order Boltzmann equation for temperature. They contribute at the level of $\sim 5-10 \%$ to the effective heating rate close to the recombination epoch at $z \simeq 10^{3}$ (Chluba et al. 2012b).

At lower redshifts, baryons and photons develop relative velocity and second order Doppler effect also contributes to the $y$ distortions and appears above in the gauge invariant combination with photon dipole. This effect can thus be considered as the mixing of dipole in the electron rest frame but in a general frame like conformal Newtonian gauge it originates in the Compton collision term ( $\mathrm{Hu}$ et al. 1994b). This is the only significant contribution from the second order Compton collision term to the spectral distortions (in addition of course to the Kompaneets term). This term can also be easily obtained by taking the part of the second order Compton collision term (see for example $\mathrm{Hu}$ et al. 1994b; Bartolo et al. 2007; Pitrou 2009) with the $y$-type spectral dependence. Higher order corrections originating in the terms which are second order in perturbation theory and also second order in energy transfer were calculated in Chluba et al. (2012b) and shown to be negligible. Fitting formulae for $\mu$ distortions as a function of spectral index and its running for primordial adiabatic perturbations are also given in Chluba et al. (2012b). Recently isocurvature modes were considered by Dent et al. (2012), Chluba et al. (2012a) have calculated the distortions from some exotic models for small-scale power spectrum, Pajer \& Zaldarriaga (2012) have pointed out the possibility of constraining non-gaussianity using $\mu$ distortions, and Ganc \& Komatsu (2012) have investigated the consequences of modified initial state for single field inflation.

Equation (20) is explicitly gauge invariant and is recommended for calculations of distortions instead of taking the time derivative of monopole, Eq. (18). In particular, Eq. (18) is accurate only in the $\mu$-era $\left(z \gtrsim 10^{5}\right)$, and the early stages of the $y$-era $\left(z \gtrsim 10^{4}\right)$, when only the shear viscosity (quadrupole) term contributes, and cannot be used to estimate the $y$-type distortions created at late times, around and after recombination. In the $y$-era ( $z \lesssim 10^{4}$ ), thermal conductivity (dipole/velocity) term and $\ell>2$ anisotropies contribute at a significant level and the full Eq. (20) must be used.

The first three terms in Eq. (20) give the dominant contribution to the dissipation of sound waves. The first term mixes the blackbodies in the dipole resulting in transfer of heat along the temperature gradient, and can thus be identified as the effect of thermal conductivity. The second term in Eq. (20), similarly, mixes the blackbodies in the quadrupole or the shear stress in the photon fluid and can thus be identified as the effect of shear viscosity. The third term takes into account the polarization

7 Our convention is same as that of (Ma \& Bertschinger 1995; Dodelson 2003) but differs from that of Zaldarriaga \& Harari (1995) by a factor of $(-i)^{\ell}$ in the definition of multipole moments. 
dependence of the Compton scattering and is a correction to the shear viscosity (Kaiser 1983). The $\ell \geq 3$ multipoles are negligible during tight coupling by definition (and thus for the $\mu$-type distortions) but give a small contribution during recombination as the tight coupling breaks down and the photons begin to free stream (Khatri et al. 2012; Chluba et al. 2012b). On substituting the conformal Newtonian gauge tight coupling solutions (Hu \& Sugiyama 1995; Zaldarriaga \& Harari 1995; Dodelson 2003), we get for the first term (ignoring the phase of the oscillations which actually differs between the left hand side and the right hand side by $\pi / 2)^{8}$,

$$
\frac{\left(3 \Theta_{1}-v\right)^{2}}{3} \approx \frac{R^{2}}{1+R} \frac{k^{2}}{\left(n_{\mathrm{e}} \sigma_{\mathrm{T}}\right)^{2}} \Theta_{1}^{2},
$$

where $R=\frac{3 \rho_{\mathrm{b}}}{4 E_{\gamma}}=\frac{693}{1+z}$ and $\rho_{\mathrm{b}}$ is the energy density of baryons. At $z>10^{5}$, when the $\mu$-type distortions are created, $R^{2} \ll 1$ and thermal conductivity contributes negligibly to the sound wave dissipation. The dominant terms during the $\mu$-type era are the second and the third (shear viscosity) terms (Zaldarriaga \& Harari 1995)

$$
\frac{9}{2} \Theta_{2}^{2}-\frac{1}{2} \Theta_{2}\left(\Theta_{2}^{\mathrm{P}}+\Theta_{0}^{\mathrm{P}}\right)=\frac{16}{15} \frac{k^{2}}{\left(n_{\mathrm{e}} \sigma_{\mathrm{T}}\right)^{2}} \Theta_{1}^{2} .
$$

If we ignore polarization, the factor of $16 / 15$ in the above equation would be replaced by $8 / 9$. At redshifts $z \gtrsim 2 \times 10^{6}$ the distortions are exponentially suppressed due to the combined action of bremsstrahlung and double Compton, which can create and destroy photons at low frequencies, and comptonization, which redistributed the photons over the entire spectrum creating a Bose-Einstein spectrum. The rate of energy injection in Eq. (20) therefore has to be multiplied by a suppression factor or blackbody visibility, $\mathcal{G}(z)$, for $z \gtrsim 10^{5}$, giving the part of the energy injection which is actually observed as $\mu$-type distortion. An analytic solution for $G(z)$ was calculated by Sunyaev \& Zeldovich (1970c), who only considered bremsstrahlung. Their solution was later applied to double Compton scattering by Danese \& de Zotti (1982) (accurate to 5-10\%) and improved recently to sub-percent accuracy in Khatri \& Sunyaev (2012). Numerical computation of the spectral distortions is possible using numerical codes such as KYPRIX (Procopio \& Burigana 2009) and CosmoTherm ${ }^{9}$ (Chluba \& Sunyaev 2012), the later code includes the energy injection due to Silk damping and is able to calculate such small distortions at high precision.

\section{Conclusions}

Mixing of blackbody spectra results in a photon distribution which is no longer a perfect blackbody but contains a $y$-type spectral distortion. The mixed spectrum has higher entropy than the average entropy of initial spectra as expected from an irreversible process which creates disorder. The energy which goes into the spectral distortion and the increase of entropy can be calculated very simply using statistical physics. The increase in entropy is, at first order in small distortions, independent of the shape of the distortion and can be calculated using the equilibrium thermodynamics formula for small addition of heat, $\mathrm{d} S=\mathrm{d} Q / T$. The part of the energy which sources distortions

\footnotetext{
8 This does not introduce any error in the calculation of heating of the average $\mathrm{CMB}$ spectrum since we should average the sound wave over a whole oscillation.

${ }^{9}$ http://www.chluba.de/CosmoTherm
}

is only $1 / 3$ of the total energy available in temperature perturbations (with respect to a blackbody at average initial temperature) and also results in increase of entropy. The remaining $2 / 3$ of the energy in perturbations goes into increasing the average blackbody temperature of the photons and can be identified with entropy conserving part of the mixing process. We have proven explicitly in this paper that the comptonization of the initial spectrum with $y$-type distortion to the Bose-Einstein spectrum does not change this 2:1 division of the dissipated energy into a blackbody part and a distortion part. From an observational point of view, we are just interested in the value of the observable $\mu$, and this 2:1 division of dissipated energy allows us to compute the value of $\mu$ in a straightforward way. $\mu$-distortions are unique and very important because it is impossible to create them after $z \lesssim 10^{5}$ and thus probe the physics of the early Universe unambiguously. $y$-type distortions on the other hand are created throughout the later history of the Universe, and it is difficult to separate the contributions from the different epochs.

We have an almost perfect blackbody in the Universe in the form of CMB. We apply our results to mixing of blackbodies in the observed CMB sky. As a result of this mixing, the spectrum observed by a telescope with finite beam size would have inevitable $y$-type distortions. This effect must be taken into account in experiments aiming to measure $\mathrm{CMB}$ spectrum at high precision. A very important application of physics of the mixing blackbodies is in the early Universe. Before recombination, the tightly coupled baryon-electron-photon plasma is excited by primordial perturbations in energy density, resulting in standing sound waves. The photons in different phases of the sound wave have a blackbody spectrum with different temperature. Photon diffusion and isotropization of the radiation field by Thomson scattering mixes these blackbodies on scales corresponding to diffusion length. These spectral distortions measure the primordial spectrum on very small scales (with the smallest scales completely inaccessible by any other means), at comoving wavenumbers $10 \lesssim k \lesssim 10^{4} \mathrm{Mpc}^{-1}$, and will thus provide a powerful new tool to constrain early Universe physics in the future. We have derived the energy release resulting from the damping of these sound waves, and the corresponding spectral distortions of the $\mathrm{CMB}$, in a simple manner using the physics of mixing of blackbodies. These results and additional (but negligible) corrections were calculated recently using second order perturbation theory in Chluba et al. (2012b). The results, for the very important $\mu$ type distortions, coincidentally are close to the estimates used in literature until now, with the main difference being a correction factor of $3 / 4$.

\section{References}

Bartolo, N., Matarrese, S., \& Riotto, A. 2007 [arXiv:astro-ph/0703496]

Bennett, C. L., Banday, A. J., Gorski, K. M., et al. 1996, ApJ, 464, L1

Chluba, J., \& Sunyaev, R. A. 2004, A\&A, 424, 389

Chluba, J., \& Sunyaev, R. A. 2006, A\&A, 458, L29

Chluba, J., \& Sunyaev, R. A. 2012, MNRAS, 419, 1294

Chluba, J., Erickcek, A. L., \& Ben-Dayan, I. 2012a, ApJ, submitted [arXiv: 1203.2681]

Chluba, J., Khatri, R., \& Sunyaev, R. A. 2012b, MNRAS, submitted [arXiv: 1202.0057$]$

Daly, R. A. 1991, ApJ, 371, 14

Danese, L., \& de Zotti, G. 1982, A\&A, 107, 39

Dent, J. B., Easson, D. A., \& Tashiro, H. 2012 [arXiv: 1202 .6066]

Dodelson, S. 2003, Modern cosmology (Amsterdam: Academic Press)

Fixsen, D. J., \& Mather, J. C. 2002, ApJ, 581, 817

Fixsen, D. J., Cheng, E. S., Gales, J. M., et al. 1996, ApJ, 473, 576

Ganc, J., \& Komatsu, E. 2012 [arXiv: 1204 .4241]

Hu, W., \& Sugiyama, N. 1995, ApJ, 444, 489

Hu, W., Scott, D., \& Silk, J. 1994a, ApJ, 430, L5 
A\&A 543, A136 (2012)

Hu, W., Scott, D., \& Silk, J. 1994b, Phys. Rev. D, 49, 648

Illarionov, A. F., \& Sunyaev, R. A. 1975, SvA, 18, 691

Jarosik, N., Bennett, C. L., Dunkley, J., et al. 2011, ApJS, 192, 14

Kaiser, N. 1983, MNRAS, 202, 1169

Khatri, R., \& Sunyaev, R. A. 2012 [arxiv: 1203.2601

Khatri, R., Sunyaev, R. A., \& Chluba, J. 2012, A\&A, 540, A124

Kogut, A., Fixsen, D. J., Chuss, D. T., et al. 2011, J. Cosmol. Astropart. Phys., 025

Komatsu, E., Smith, K. M., Dunkley, J., et al. 2011, ApJS, 192, 18

Kompaneets, A. S. 1956, Zh. Eksp. Teor. Fiz., 31, 876

Landau, L. D., \& Lifshitz, E. M. 1980, Statistical physics, Part. 1 (Oxford: Butterworth-Heinemann)

Lifshitz, E. M. 1946, J. Phys. (USSR), 10, 116

Ma, C.-P., \& Bertschinger, E. 1995, ApJ, 455, 7
Pajer, E., \& Zaldarriaga, M. 2012 [arXiv: 1201.5375]

Peebles, P. J. E., \& Yu, J. T. 1970, ApJ, 162, 815

Pitrou, C. 2009, Class. Quant. Grav., 26, 065006

Procopio, P., \& Burigana, C. 2009, A\&A, 507, 1243

Rubiño-Martín, J. A., Chluba, J., \& Sunyaev, R. A. 2008, A\&A, 485, 377

Silk, J. 1968, ApJ, 151, 459

Sunyaev, R. A., \& Zeldovich, Y. B. 1970a, Ap\&SS, 9, 368

Sunyaev, R. A., \& Zeldovich, Y. B. 1970b, Ap\&SS, 7, 3

Sunyaev, R. A., \& Zeldovich, Y. B. 1970c, Ap\&SS, 7, 20

Weinberg, S. 1971, ApJ, 168, 175

Zaldarriaga, M., \& Harari, D. D. 1995, Phys. Rev. D, 52, 3276

Zeldovich, Y. B., \& Sunyaev, R. A. 1969, Ap\&SS, 4, 301

Zeldovich, Y. B., Illarionov, A. F., \& Sunyaev, R. A. 1972, Sov. J. Exper. Theor. Phys., 35, 643 